\title{
Effects of PDE-5 Inhibition on the Cardiopulmonary System After 2 or 4 Weeks of Chronic Hypoxia
}

\author{
Coline Nydegger ${ }^{1} \cdot$ Antonio F. Corno $^{2} \cdot$ Ludwig K. von Segesser $^{3} \cdot$ Maurice Beghetti $^{4,5} \cdot$ Michele Samaja $^{6}$. \\ Giuseppina Milano ${ }^{1}$
}

Published online: 1 July 2019

(C) The Author(s) 2019

\begin{abstract}
Purpose In pulmonary hypertension (PH), hypoxia represents both an outcome and a cause of exacerbation. We addressed the question whether hypoxia adaptation might affect the mechanisms underlying PH alleviation through phosphodiesterase-5 (PDE5) inhibition. Methods Eight-week-old male Sprague-Dawley rats were divided into two groups depending on treatment (placebo or sildenafil, a drug inhibiting PDE5) and were exposed to hypoxia $\left(10 \% \mathrm{O}_{2}\right)$ for $0(t 0, n=9 / 10), 2(t 2, n=5 / 5)$ or $4(t 4, n=5 / 5)$ weeks. The rats were treated $(0.3 \mathrm{~mL}$ i.p.) with either saline or sildenafil $(1.4 \mathrm{mg} / \mathrm{Kg}$ per day).

Results Two-week hypoxia changed the body weight ( $-31 \%$ vs. $-27 \%$, respectively, $P=\mathrm{NS})$, blood hemoglobin $(+25 \%$ vs. + $27 \%, P=\mathrm{NS})$ and nitrates+nitrites $(+175 \%$ vs. $+261 \%, P=0.007)$, right ventricle fibrosis $(+814 \%$ vs. $+317 \%, P<0.0001)$, right ventricle hypertrophy $(+84 \%$ vs. $+49 \%, P=0.007)$ and systolic pressure $(+108 \%$ vs. $+41 \%, P=0.001)$, pulmonary vessel density ( $+61 \%$ vs. $+46 \%, P=\mathrm{NS})$, and the frequency of small $(<50 \mu \mathrm{m}$ wall thickness $)$ vessels $(+35 \%$ vs. $+13 \%, P=0.0001)$. Most of these changes were maintained for 4-week hypoxia, except blood hemoglobin and right ventricle hypertrophy that continued increasing ( $+52 \%$ vs. $+42 \%, P=\mathrm{NS}$; and $+104 \%$ vs. $+83 \%, P=0.04)$. To further assess these observations, small vessel frequency was found to be linearly related with the right ventricle-developed pressure independent of hypoxia duration. Conclusions Thus, although hypoxia adaptation is not yet accomplished after 4 weeks, PH alleviation by PDE5 inhibition might nevertheless provide an efficient strategy for the management of this disease.
\end{abstract}

Keywords Chronic hypoxia Adaptation $\cdot$ Phosphodiesterase-5 inhibition $\cdot$ Pulmonary angiogenesis $\cdot$ Right-ventricle hypertrophy

Giuseppina Milano

Giuseppina.Milano@chuv.ch

1 Department Cœur-Vaisseaux, Cardiac Surgery center, University Hospital of Lausanne, Lausanne, Switzerland

2 Cardiovascular Research Center, University of Leicester, Leicester, UK

3 Cardiovascular Research Unit, University Hospital of Lausanne, Lausanne, Switzerland

4 Pediatric Cardiology Unit, University of Geneva, Geneva, Switzerland

5 Centre Universitaire Romand de Cardiologie et Chirurgie Cardiaque Pédiatrique, Children's University Hospitals, Geneva and Lausanne, Lausanne, Switzerland

6 Department of Health Science, University of Milan, Milan, Italy

\section{Introduction}

The pathophysiology of pulmonary hypertension $(\mathrm{PH})$ includes progressive rising of the pulmonary artery pressure, with subsequent straining of the right ventricle (RV) thereby causing hypertrophy and leading to potential right heart failure and/or ventricular arrhythmias. In addition to the fact that PH contributes to the impairment of the pulmonary function with subsequent deterioration of the gas exchange, thereby causing systemic hypoxia, hypoxia per se also represents one of the main complications of $\mathrm{PH}$, establishing an irreversible vicious circle, whereby PH causes hypoxia, which in turn exacerbates PH [1]. Healthy individuals, however, may in part compensate systemic hypoxia through adaptation, a complex phenomenon recruiting the pulmonary, cardiovascular, hematological, metabolic, and endocrine systems $[2,3]$. Is the mechanism of hypoxia adaptation also applicable in patients with $\mathrm{PH}$, caused either by congenital heart defects or by acquired diseases? This question 
might be relevant because a therapy proven to be effective in the treatment of PH, e.g., phosphodiesterase-5 (PDE5) inhibition by sildenafil $[4,5]$, generally used as a vasodilator agent to antagonize the vasoconstriction present in pulmonary hypertension, could be extended to treat also the negative effects of pulmonary hypertension such as subsequent hypoxia. Therefore, it is important to assess whether PH-induced hypoxia is also responding to adaptation. Remarkably, while studies have been reported on the long-term effects in the pediatric population with $\mathrm{PH}$ [6-9], evidence for similar long-term beneficial effects of PDE5 inhibitors in COPD patients is still controversial [10-12], thereby justifying experimental studies aiming to answer the question whether the effects of hypoxia, and hence of PDE5 inhibition, are time-dependent.

The design of the present study was focused on assessing if some of the mechanisms known to underlie alleviation of hypoxia-induced PH by PDE5 inhibition [13] are time-dependent. Such information might be important not only to confirm the viability of the mechanisms for a better treatment of hypoxia of longer duration but also to assess whether other timedependent mechanisms, potentially linked to hypoxia adaptation, may eventually override the protection afforded by sildenafil and introduce bias in the interpretation of results. Therefore, the target of this study was to test whether the cardiopulmonary dysfunction caused by hypoxia and the correction provided by sildenafil display time-dependent features. To this purpose, we examined two time durations ( 2 and 4 weeks) of challenge (hypoxia) and treatment (sildenafil).

\section{Methods}

\section{Protocol}

We used male 8-week old Sprague-Dawley rats (200-250 g initial nominal weight). Rats were randomly divided into two groups depending on treatment (without/with sildenafil) and were exposed to hypoxia $\left(10 \% \mathrm{O}_{2}\right)$ as described previously [13] for $0(t 0, n=9 / 10), 2(t 2, n=5 / 5)$, or $4(t 4, \mathrm{n}=5 / 5)$ weeks. Rats were treated $(0.3 \mathrm{~mL}$ i.p. $)$ with either saline or sildenafil $(1.4 \mathrm{mg} / \mathrm{kg} / \mathrm{day})$. Treatments started in correspondence to exposure to hypoxia.

Twenty-four hours after the last treatment, rats were anesthetized ( $80 \mathrm{mg} / \mathrm{kg}$ xylazine, $100 \mathrm{mg} / \mathrm{kg}$ ketasol, and $1500 \mathrm{IU}$ heparin i.p.) in the compensation chamber at $10 \% \mathrm{O}_{2}$.

To assess the hemodynamics, anesthetized rats were placed on a heating pad at $37^{\circ} \mathrm{C}$ and ventilated at 50 cycles $\min ^{-1}$ (tidal volume of $2.5 \mathrm{~mL}$, Harvard Apparatus model 683, Holliston, MA, USA) with either room air $\left(21 \% \mathrm{O}_{2}\right)$ or hypoxic $\left(10 \% \mathrm{O}_{2}\right)$ atmosphere for normoxic and hypoxic groups, respectively. To evaluate the LV pressures, a Millar catheter was introduced into the LV via the right carotid artery. To evaluate RV pressures, the chest cavity was opened and
Millar catheter was directly introduced in the RV cavity using a 24-gauge needle, and connected to a MPVS Ultra system (Millar Instruments) to record the pressure waves. At the end of hemodynamic analysis, lungs and hearts were perfused enblock with PBS via the RV with efflux through a small opening in the left atrium [14], placed in PBS and kept on ice. Atria were removed, the RV and the septum (S) were isolated, blotted dry and weighed to obtain the $\mathrm{RV} /(\mathrm{LV}+\mathrm{S})$ ratio.

\section{Pulmonary Vascular Remodeling}

The degree of muscularization of pulmonary arterioles was assessed from immunohistochemical staining of the small pulmonary artery, as previously described [13]. Briefly, the lungs were inflated with $10 \%$ formalin at $25 \mathrm{~cm} \mathrm{H}_{2} \mathrm{O}$ pressure through the trachea for $15 \mathrm{~min}$, excised and finally processed for paraffin embedding. Paraffin-embedded lungs were serially sectioned at $8-\mu \mathrm{m}$ thickness. Following citrate-based antigen retrieval, the sections were blocked with $5 \%(v / v)$ goat serum for $1 \mathrm{~h}$. Then, sections were incubated with an antibody against smooth muscle $\alpha$-actin ( $\alpha$-SMA 1:250, clone 1A4, Sigma-Aldrich) overnight at $4{ }^{\circ} \mathrm{C}$, followed by a goat antimouse IgG secondary antibody (1/500, DAKO), developed with 3,3'-diaminobenzidine, and counter stained with hematoxylin and eosin. All transversal cut arterioles were quantitatively analyzed at $\times 40$ magnification using an images analysis system Nikon eclipse $80 \mathrm{i}$ camera and NIH image software (Nikon Instruments Inc., Melville, NY, USA). Pulmonary arterial thickening was assessed by calculating the percent pulmonary artery thickness using the following formula: $100 \times$ (perivascular area-luminal area)/luminal area. Pulmonary arteries were categorized as follows: $<50 \mu \mathrm{m}$, 50-100 $\mu \mathrm{m}, 100-200 \mu \mathrm{m}$, and $>200 \mu \mathrm{m}$. Ten vessels were analyzed for each rat, in six rats per group per time-point. Morphological analyses were conducted in a double-blind method.

\section{RV Fibrosis}

RV fibrosis was evaluated by routine Masson's staining. The images were taken in random field at a magnification of $\times 200$, and the area of cardiac fibrosis was calculated by using NIH image software.

\section{Biochemical Measurements}

Standard Western blotting analysis was performed in lung and cardiac lysates [14] using antibodies against p-eNOS (Ser ${ }^{1177}$, 1:1000, Cell Signaling Technology) and e-NOS (1:1000, Santa Cruz Biotechnology, Santa Cruz). Secondary HRPconjugated antibodies were applied for $1 \mathrm{~h}$ at room temperature, and signals were detected by using the enhanced chemiluminescence system (Amersham, Arlington Heights, IL, 
USA) of a commercial ECL kit. Quantification of the band intensities was performed using NIH ImageJ.

Blood hemoglobin ( $\mathrm{Hb}$, Drabkin's reagent) and plasma nitrates and nitrites (NOx, colorimetric Griess reaction) were measured in heparinized samples taken after euthanasia.

\section{Statistics}

Data are expressed as mean \pm SEM. To measure the effect of hypoxia duration, we performed one-way analysis of variance followed by the Bonferroni test to detect significant differences between the time points. To measure the differences between the two treatments at every time point, we used the unpaired $t$ test. The significance level was set at $P=0.05$.

\section{Results}

All the animals used in this study survived the protocols. Hypoxia markedly decreased the body weight at $t 2$ irrespectively of sildenafil treatment $(P<0.05 \mathrm{vs}$. $t 0$ for both control and sildenafil, Fig. 1). This change was maintained at $t 4(P=$ NS vs. $t 2$ in both groups). A slight favorable effect of sildenafil was, however, noticed at $t 4(P=0.046)$. Hypoxia steadily increased blood [Hb] at both $t 2(P<0.05$ vs. $t=0)$ and $t 4$ ( $P<0.05$ vs. $t=2$ weeks $)$. A slight borderline effect of sildenafil was observed at $t 4(P=0.052)$.

At $t 2$, the heart/body weight ratio increased by $60 \%$ in controls $(P<0.05$ vs. $t 0)$ and did not change further at $t 4$ ( $P=$ NS vs. $t=2$ weeks), indicating no increase in myocardial hypertrophy after 2 -week hypoxia. Sildenafil halved the progression of myocardial hypertrophy at both times $(P=0.0035$ and $P<0.00001$ vs. control at $t 2$ and $t 4$, respectively). To follow up the effect on RV hypertrophy, we used the RV/ $(\mathrm{LV}+\mathrm{S})$ ratio. This index increased markedly at $t 2(P<0.05$ vs. $t 0$ for both control and sildenafil), but the increase was less marked in sildenafil than controls $(P=0.007)$. At $t 4$, the RV/ $(\mathrm{LV}+\mathrm{S})$ ratio did not change appreciably in control hearts $(P=$ NS vs. $t 2)$ but increased significantly in sildenafil hearts $(P<0.05$ vs $t 2)$. Consequently, the difference between control and sildenafil decreased but remained significant $(P=0.046)$.

Two-week hypoxia augmented the vessel density in both the right and left lungs (only the left lung is shown for clarity, $P<0.05$ vs. $t 0)$. Only a borderline $(P=0.058)$ effect of

\section{Final body weight, $g$}

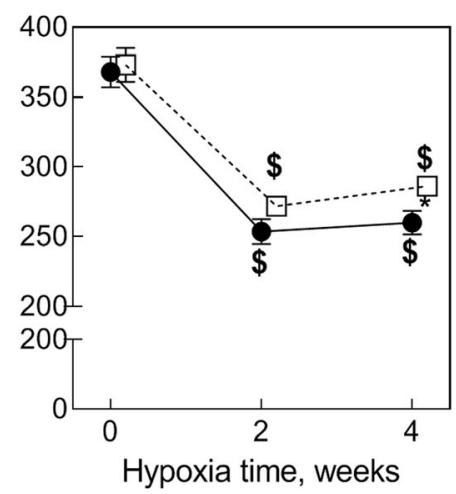

Right/Left+Septum

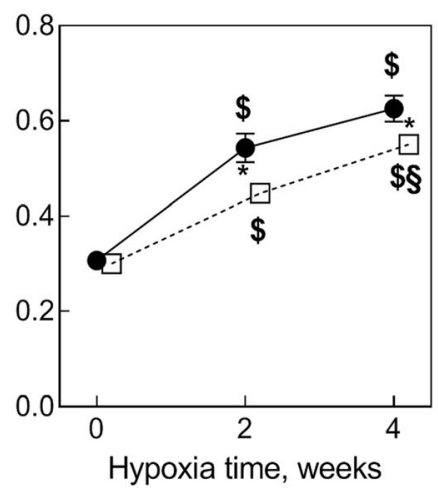

Blood Hb, g/L

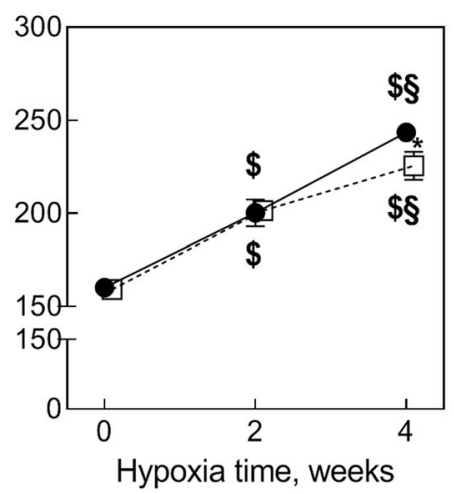

Vessels in left lung

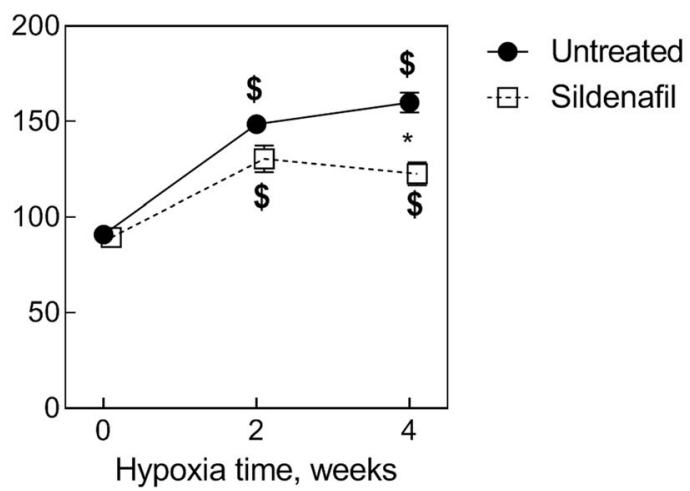

Fig. 1 Effects of various durations of hypoxia in the absence (filled circles) and in the presence (empty squares) of sildenafil $(1.4 \mathrm{mg} / \mathrm{kg} /$ day). The vertical bars represent the SEM. Sildenafil data points are slightly nudged to the right to enable distinguishing overlapping
Heart/body weight, $\mathrm{mg} / \mathrm{g}$

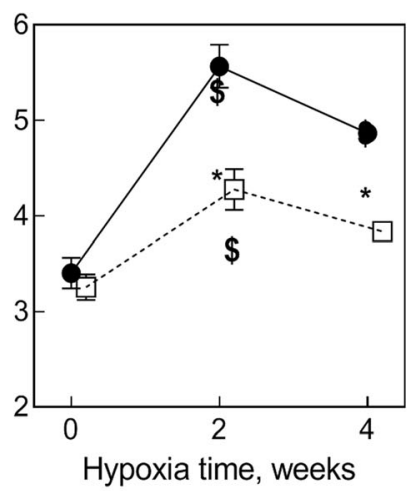

Hypoxia time, weeks symbols and error bars. $\$, \S P<0.05$ (ANOVA and Bonferroni tests) with respect to $t 0$ and $t 2$, respectively. $* P<0.05$ (unpaired Student's $t$ test) between control and sildenafil groups for each time point 
Fig. 2 Hemodynamic parameters. Effects of various durations of hypoxia in the absence (filled circles) and in the presence (empty squares) of sildenafil $(1.4 \mathrm{mg} / \mathrm{kg} /$ day). The vertical bars represent the SEM. Sildenafil data points are slightly nudged to the right to enable distinguishing overlapping symbols and error bars. $\$, \S P<$ 0.05 (ANOVA and Bonferroni tests) with respect to $t 0$ and $t 2$, respectively. $* P<0.05$ (unpaired Student's $t$ test) between control and sildenafil groups for each time point
Pressures, $\mathrm{mmHg}$

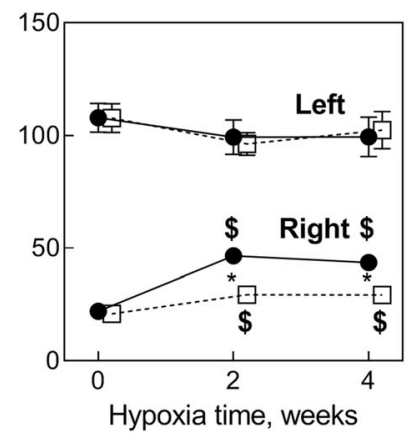

End-diastolic pressure, $\mathrm{mmHg}$

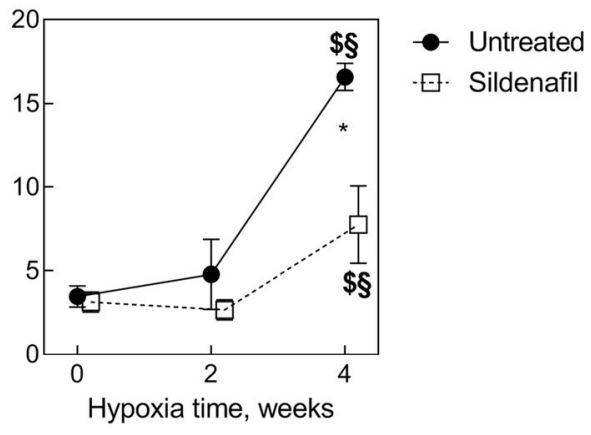

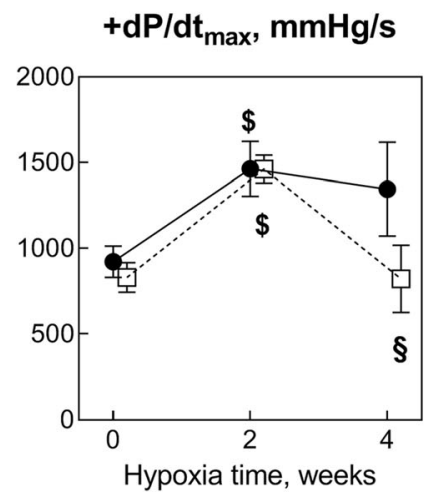

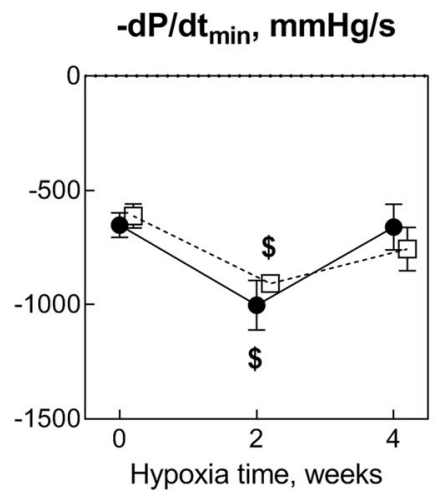

sildenafil was observed. At $t 4$, the vessel density did not change appreciably vs. $t 2$, but the anti-angiogenic effect of sildenafil became very clear $(P<0.0001)$.

Figure 2 shows several hemodynamic parameters. The LV systolic pressure remained unaffected by hypoxia ( $P=\mathrm{NS}$ vs. t0) and sildenafil $(P=\mathrm{NS})$. By contrast, the RV systolic pressure nearly doubled at $t 2$ in controls $(P<0.05$ vs. $t 0)$, indicative of $\mathrm{PH}$, with no change at $t 4$. In sildenafil rats at $t 2$, the raise in $\mathrm{RV}$ systolic pressure was markedly less $(+41 \%$ vs. $+108 \%$ in controls, $P=0.0014)$ and was maintained at $t 4(+40 \%$ vs. $+97 \%$, $P=0.002)$. The end-diastolic pressure did not change appreciably at $t 2$, but it increased significantly at $t 4$. The increase was + $377 \%(P<0.0001)$ and $+147 \%(P=0.02)$ in control and sildenafil rats, respectively, with a highly significant difference between the two groups $(P=0.007)$. By contrast, $+\mathrm{dP} / \mathrm{dt}_{\max }$ and $-\mathrm{dP} / \mathrm{dt}_{\max }$ were significantly increased $+50-70 \%$ at $t 2$ in both groups with no significant difference. At $t 4$, sildenafil blunted the increase in $+\mathrm{dP} / \mathrm{dt}_{\max }$ vs. no significant effects in $-\mathrm{dP} / \mathrm{dt}_{\max }$.

Figure 3 shows the frequency of lung vessels belonging to four categories of wall thickness, arbitrarily divided into small $(0-50 \mu \mathrm{m})$, medium $(50-100 \mu \mathrm{m})$, large (100-200 $\mu \mathrm{m})$, and very large $(>200 \mu \mathrm{m})$. Because data from right lungs are indistinguishable from those from left lungs (not shown), for clarity, we report only data from left lungs. It appears that the effect of 2-week hypoxia was much pronounced for small vessels and progressively diminished for increasing vessel diameter. For example, the number of vessels in the 0$50 \mu \mathrm{m}$ range increased by $35 \%$ vs. $11 \%$ for $50-100 \mu \mathrm{m}$, and near zero for larger vessels. As a result, the effect of sildenafil was more marked in small than in large vessels, $13 \%$ in the 0 $50 \mu \mathrm{m}$ range $(P<0.0001 \mathrm{vs}$. control $)$ and virtually zero for larger vessels. In no case, however, was there a difference between $t 2$ and $t 4$, indicating a stable situation.

Figure 4 shows indexes of RV fibrosis obtained as explained in the "Methods" section. While hypoxia increased fibrosis $(P<0.0001)$, the increase was markedly more pronounced in control vs. sildenafil rats $(P=0.007$ and 0.0005 at $t 2$ and $t 4$, respectively), indicating that sildenafil has a strong anti-fibrotic effect in this model.

Figure 5 shows that the p-eNOS/eNOS ratio highlights the activation of endothelium NO-producing enzymes in the heart and in the lung. In both cases, 2-week hypoxia decreased the p-eNOS/eNOS ratio in control $(P<0.05)$, as opposite to constant and increased $\mathrm{p}$-eNOS/eNOS ratio in sildenafil-treated hearts $(P=0.01)$ and lungs $(P<0.0001)$, respectively. At $t 4$, the situation remained stable, highlighting a protective effect of sildenafil in both tissues independent of hypoxia duration.

Plasma NOx concentration marks the blood NO storage level. Two-week hypoxia increased this marker in both control and sildenafil rats $(P<0.05$ vs. $t 0)$, but in sildenafil-treated rats, this effect was higher $(P=0.007)$. The situation did not change appreciably at $t 4$.

To further assess the effects observed above, Fig. 6 shows the relationship between the frequency of small vessels $(0-50 \mu \mathrm{m})$ and the pressure developed by LV (upper panel) and RV (lower panel). As far as the LV is 
Wall thickness $0-50 \mu \mathrm{m}, \%$

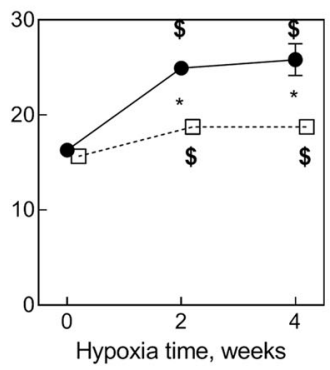

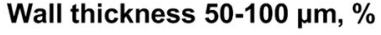

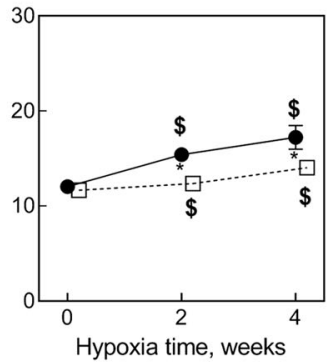

Wall thickness $100-200 \mu \mathrm{m}, \%$

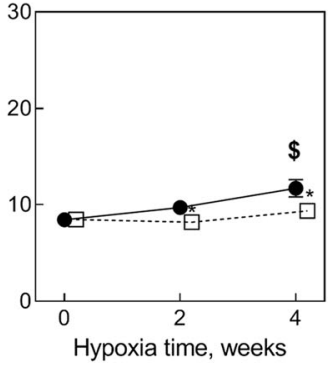

Wall thickness $>200 \mu \mathrm{m}, \%$

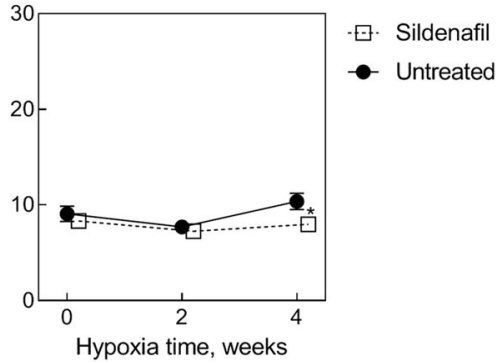

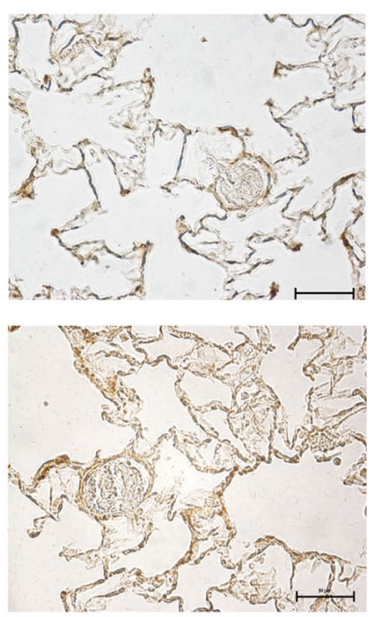

$\mathbf{t}=\mathbf{0}$ weeks

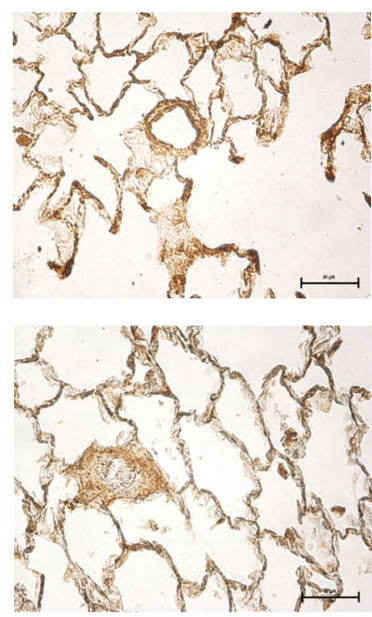

$\mathbf{t}=\mathbf{2}$ weeks
Fig. 3 Effects of various durations of hypoxia in the absence (filled circles) and in the presence (empty squares) of sildenafil $(1.4 \mathrm{mg} / \mathrm{kg} /$ day). The vertical bars represent the SEM. Sildenafil data points are slightly nudged to the right to enable distinguishing overlapping symbols and error bars. $\$, \S P<0.05$ (ANOVA and Bonferroni tests) with respect to $t 0$ and $t 2$, respectively. $* P<0.05$ (unpaired Student's $t$ test) between control and sildenafil groups for each time point. The microphotographs show representative examples of the Masson's staining to evaluate lung tissue fibrosis in the various cases considered in this study concerned, the slope of the best-fit line $(-1.357 \pm 0.9287)$ did not differ from zero $(P=\mathrm{NS})$. However, the best-fit slope increased markedly to $+2.352 \pm 0.2563(P<0.0001$ vs. zero) in the RV. Of interest, the relationship between the RV systolic pressure and the small vessels frequency was independent of hypoxia duration, indicating that the treatment with sildenafil could reduce the RV systolic pressure for both time durations.

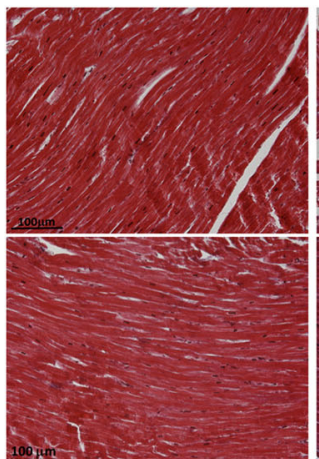

$\mathrm{t}=\mathbf{0}$ weeks

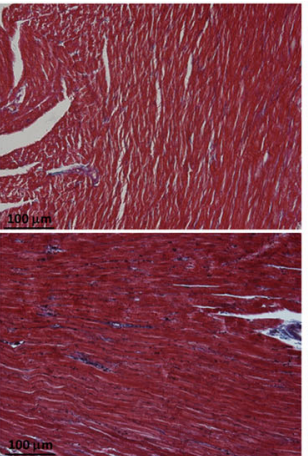

$\mathrm{t}=2$ weeks

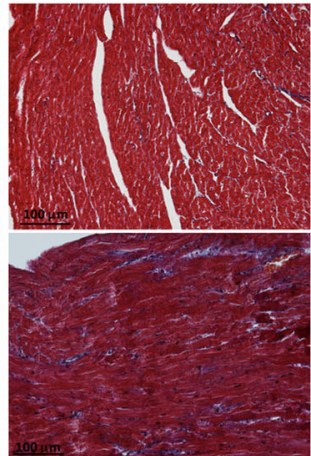

$\mathbf{t}=4$ weeks

Fig. 4 Effects of various durations of hypoxia in the absence (filled circles) and in the presence (empty squares) of sildenafil $(1.4 \mathrm{mg} / \mathrm{kg} /$ day) on RV cardiac fibrosis. The vertical bars represent the SEM. Sildenafil data points are slightly nudged to the right to enable distinguishing overlapping symbols and error bars. $\$, \S P<0.05$

\section{RV Fibrosis, \% positive area}

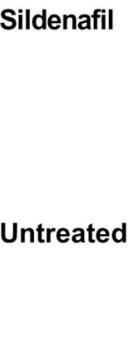

(ANOVA and Bonferroni tests) with respect to $t 0$ and $t 2$, respectively. $* P<0.05$ (unpaired Student's $t$ test) between control and sildenafil groups for each time point. The microphotographs show representative examples of the Masson's staining to evaluate tissue fibrosis in the various cases considered in this study 


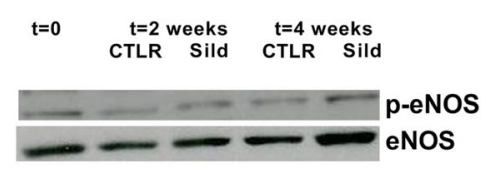

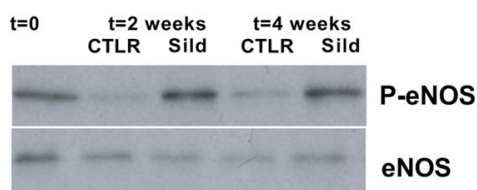

eNOS
p-eNOS / eNOS, heart

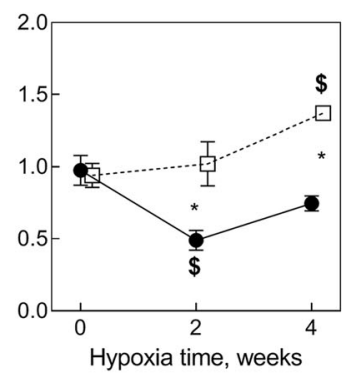

p-eNOS / eNOS, lung

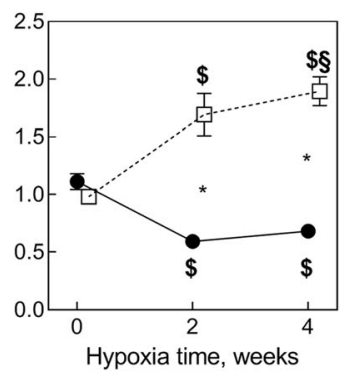

NOx, mM

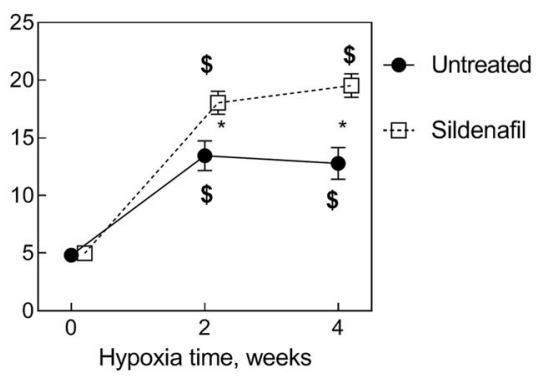

Fig. 5 Effects of various durations of hypoxia in the absence (filled circles) and in the presence (empty squares) of sildenafil $(1.4 \mathrm{mg} / \mathrm{kg} /$ day). The vertical bars represent the SEM. Sildenafil data points are slightly nudged to the right to enable distinguishing overlapping

\section{Discussion}

In our observations, 2- and 4-week duration of hypoxia induced relevant non-lethal cell and molecular alterations. As expected, hypoxia impaired the homeostasis and increased
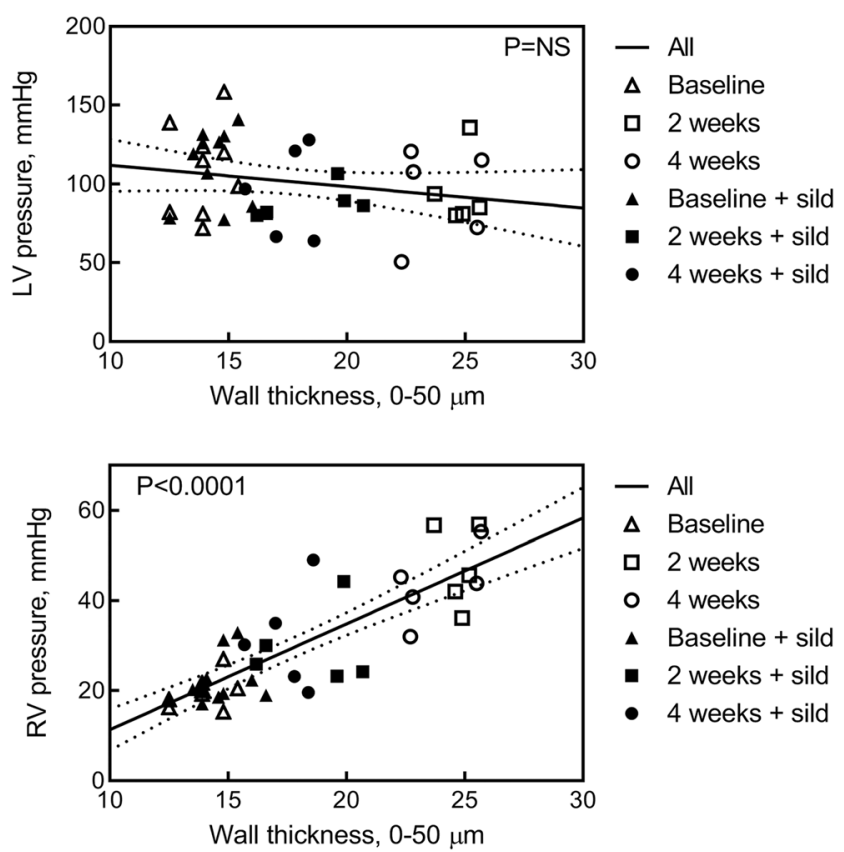

Fig. 6 Relationship between the frequency of small diameter vessels (0$50 \mu \mathrm{m}$ ) and the pressure developed by the left (upper panel) and right (lower panel) ventricle for each experimental data point. Empty and filled symbols refer to rats treated with placebo and sildenafil, respectively. Triangles, squares, and circles represent data taken at $t 0, t 2$, and $t 4$, respectively. The best-fit lines and the 95\% confidence limits referring to all available data points are shown. The values of the slope are -1.357 \pm 0.9287 and $+2.352 \pm 0.2563$ for the left and right ventricle, respectively $(P=\mathrm{NS}$ and $P<0.0001$ vs. zero) symbols and error bars. $\$, \S P<0.05$ (ANOVA and Bonferroni tests) with respect to $t 0$ and $t 2$, respectively. $* P<0.05$ (unpaired Student's $t$ test) between control and sildenafil groups for each time point. Representative Western blots are shown

blood [Hb] and NOx, and increased the degree of RV hypertrophy, the extent of pulmonary angiogenesis, the total number of small vessels in the lungs, and the values of RV systolic pressures, without any direct or secondary influence on LV pressures. Most, but not all of these changes, occur within 2 weeks and are essentially maintained unchanged at the 4week interval. However, some of the variables investigated, including blood [Hb] and RV hypertrophy, continue to change with the extension of the duration of hypoxia from 2 to 4 weeks. Whereas augmenting $[\mathrm{Hb}]$ reflects a situation of ongoing hypoxia without complete adaptation, increased RV hypertrophy reflects the long-term morphological effects of pro-angiogenic and proliferative factors engaged during the first 2 weeks of hypoxia. Thus, although adaptation seems to be a process not yet completely accomplished after 4-week hypoxia, most of the myocardial and pulmonary responses to hypoxia are independent from the total process of adaptation.

NO handling is of critical relevance because NO controls myriads of cell/molecular pathways relevant for heart and lung viability. For example, RV hypertrophy without lung hypertrophy, as well as the raise of RV pressure, without effects on LV pressure, has been associated with increased eNOS phosphorylation in both hearts and lungs, and increased plasma NOx levels [13]. Remarkable blunting of all these features through PDE5 inhibition by sildenafil represents a solid proof-of-concept for the employment of this drug to alleviate symptoms in several cardiopulmonary diseases including PH [4] and to correct hypoxia-induced RV hypertrophy [1]. This scenario reflects a situation where sildenafilinduced increase in cGMP levels upregulates eNOS phosphorylation [15-20], and hence plasma NOx, which translates into cardiomyocyte apoptosis mitigation. In addition to its usefulness to treat heart failure without remarkable adverse 
effects [21], sildenafil may also have effects not only necessarily linked to vasodilation, as for example increased recruitment of bone marrow-derived c-kit+ cells with amelioration of pulmonary hemodynamic [14]. These mechanisms, however, have been studied within a single 2-week hypoxia situation, and there are no clues to ascertain whether such mechanisms reflect a transient situation or remain constant overtime [1]. In this study, we demonstrate that most of the underlying mechanisms are essentially unaffected by the duration of hypoxia and thus reflect a stable condition, independent from hypoxia adaptation. This is supported by the observation that the values of the pulmonary artery pressures in healthy highlanders increase in correlation with altitude and with the degree of exercise, and are related with a delayed postnatal remodeling of the distal pulmonary arterial branches [22].

The entity of the distribution of the vessels within the lungs is markedly depending on the diameter of the vessels. While the size of the small pulmonary vessels is affected by the degree of hypoxia and by sildenafil administration, the effects of both hypoxia and sildenafil are progressively diminishing in correlation with the size of the vessels. If we accept that the size of the vessel is associated with their degree of maturation, then this effect may be attributed to their maturation process. Although there is still disagreement about the molecular trigger of such process, the degree of phosphorylation of eNOS is most probably an important mediator; its persistent increase after 2 and 4 weeks of hypoxia in both the myocardial and pulmonary tissues of the sildenafil-treated rats reflects the angiogenic stimulus caused by hypoxia and the persistent correction of this stimulus by sildenafil.

The response to sildenafil, evident at both $\mathrm{t} 2$ and $\mathrm{t} 4$, can be considered to be due to the combination of the indirect effects on the RV function as a consequence of reduced afterload secondary to reduced pulmonary vascular resistance, as well as of the direct effect on the RV diastolic function. Whereas the effect of the afterload reduction was already well established [23], the capability of sildenafil to improve RV diastolic function was also previously reported in the presence of fixed afterload increase, such as that obtained by pulmonary artery banding [24]. This double action of sildenafil was confirmed by our observations, with the RV hemodynamics improved by sildenafil despite the presence of RV fibrosis at both $t 2$ and $t 4$.

With regard to the correlation between RV function and fibrosis, topic of controversies in the recent literature, it is important to evaluate presence, degree and distribution of fibrosis in the RV, as well as the duration of fibrosis. All these can influence not only RV systolic function, as generally studied and reported, but also diastolic function. Our observations, for instance, have proven the positive effects of sildenafil on RV diastolic function, regardless of the associated effects of the pulmonary vascular resistance, because the degree and distribution of RV fibrosis remained unchanged after 2 and
4 weeks. Masson staining is a reliable procedure to evaluate RV fibrosis [25]. The results shown in Figs. 2 and 4 are in essential agreement with a recent report that, although RV fibrosis is clearly linked to RV dysfunction, reduction of RV fibrosis does not directly improve cardiac function [26].

\section{Limits of the study}

As a consequence of the design of our study, the treatment with sildenafil was started simultaneously with the induction of chronic hypoxia, and we are fully aware of the potential criticism of missing the evaluation of the potential effects of sildenafil after establishing chronic hypoxia. On the other hand, the effects of sildenafil on pulmonary hypertension per se are well acknowledged. Moreover, the RV diastolic pressures, which could provide useful information in order to correlate the values of RV end-diastolic pressure with the degree of RV hypertrophy and/or of PH, were not recorded in this study because of logistic reasons.

In this study, sildenafil was not compared with other pulmonary vasodilator drugs which recently have appeared on the market, because our aim was to specifically evaluate the effects of sildenafil, without adding other confounding factors.

\section{Conclusions}

Sildenafil provides an efficient strategy for the management of $\mathrm{RV}$ hypertrophy, $\mathrm{PH}$, and pulmonary remodeling, thanks to its effects on the pulmonary vasculature, facilitating improvement of the RV function, irrespectively of hypoxia adaptation. This finding suggests that sildefanil potentially could be used in the management of clinical settings characterized by pulmonary hypertension complicated by subsequent chronic hypoxia, as in PH secondary to congenital heart defects or acquired lung issues with chronic obstructive disease. Prospective randomized clinical trials are required to verify the applicability of this therapeutic strategy in patients.

Funding The Swiss National Foundation supported this work: FN 310000-110058.

\section{Compliance with Ethical Standards}

The study was conducted in accordance with the Guide for the Care and Use of Laboratory Animals, published by the National Institutes of Health (NIH Publication No. 85-23, Revised 1996). Experimental protocols conformed to Swiss law. The local ethical committee for animal research (Service de la Consommation et des Affaire Vétérinaires, SCAV) approved the protocol (authorization VD2467).

Conflict of Interest The authors declare that they have no competing interests. 
Open Access This article is distributed under the terms of the Creative Commons Attribution 4.0 International License (http:// creativecommons.org/licenses/by/4.0/), which permits unrestricted use, distribution, and reproduction in any medium, provided you give appropriate credit to the original author(s) and the source, provide a link to the Creative Commons license, and indicate if changes were made.

\section{References}

1. Milano G, Bianciardi P, Rochemont V, Vassalli G, Segesser LK, Corno AF, et al. Phosphodiesterase-5 inhibition mimics intermittent reoxygenation and improves cardioprotection in the hypoxic myocardium. PLoS One. 2011;6(11):e27910.

2. Samaja M. Blood gas transport at high altitude. Respiration. 1997;64(6):422-8.

3. van Loon RL, Bartelds B, Wagener FA, Affara N, Mohaupt S, Wijnberg H, et al. Erythropoietin attenuates pulmonary vascular remodeling in experimental pulmonary arterial hypertension through interplay between endothelial progenitor cells and Heme oxygenase. Front Pediatr. 2015;3:71.

4. Guazzi M, Samaja M. The role of PDE5-inhibitors in cardiopulmonary disorders: from basic evidence to clinical development. Curr Med Chem. 2007;14:1893-910.

5. Siehr SL, McCarthy EK, Ogawa MT, Feinstein JA. Reported sildenafil side effects in pediatric pulmonary hypertension patients. Front Pediatr. 2015;3:12.

6. Barst RJ, Beghetti M, Pulido T, Layton G, Konourina I, Zhang M, et al. STARTS-2: long-term survival with oral sildenafil monotherapy in treatment-naive pediatric pulmonary arterial hypertension. Circulation. 2014;129(19):1914-23.

7. Negoi RI, Ghiorghiu I, Filipoiu F, Hostiuc M, Negoi I, Ginghina C. Severe pulmonary arterial hypertension associated with congenital cardiac shunts: evolution under specific treatment. J Med Life. 2017;10(2):131-8.

8. Zhou C, Guo X, Cui Q, Liu X, Su G, Zhang J. Sildenafil improves the function of endothelial cells in patients suffering from congenital heart disease with pulmonary hypertension. Pharmazie. 2016;71(10):570-4.

9. Mori H, Park IS, Yamagishi H, Nakamura M, Ishikawa S, Takigiku $\mathrm{K}$, et al. Sildenafil reduces pulmonary vascular resistance in single ventricular physiology. Int J Cardiol. 2016;221:122-7.

10. Seeger W, Adir Y, Barbera JA, Champion H, Coghlan JG, Cottin V, et al. Pulmonary hypertension in chronic lung diseases. J Am Coll Cardiol. 2013;62(25 Suppl):D109-16.

11. Dominguez-Fandos D, Valdes C, Ferrer E, Puig-Pey R, Blanco I, Tura-Ceide $\mathrm{O}$, et al. Sildenafil in a cigarette smoke-induced model of COPD in the guinea pig. Eur Respir J. 2015;46(2):346-54.

12. Vitulo P, Stanziola A, Confalonieri M, Libertucci D, Oggionni T, Rottoli P, et al. Sildenafil in severe pulmonary hypertension associated with chronic obstructive pulmonary disease: a randomized controlled multicenter clinical trial. J Heart Lung Transplant. 2017;36(2):166-74.
13. Nydegger C, Martinelli C, Di Marco F, Bulfamante G, von Segesser L, Tozzi P, et al. Phosphodiesterase-5 inhibition alleviates pulmonary hypertension and basal Lamina thickening in rats challenged by chronic hypoxia. Front Physiol. 2018;9:289.

14. Favre S, Gambini E, Nigro P, Scopece A, Bianciardi P, Caretti A, et al. Sildenafil attenuates hypoxic pulmonary remodelling by inhibiting bone marrow progenitor cells. J Cell Mol Med. 2017;21(5):871-80

15. Lepore JJ, Maroo A, Pereira NL, Ginns LC, Dec GW, Zapol WM, et al. Effect of sildenafil on the acute pulmonary vasodilator response to inhaled nitric oxide in adults with primary pulmonary hypertension. Am J Cardiol. 2002;90(6):677-80.

16. Schermuly RT, Kreisselmeier KP, Ghofrani HA, Yilmaz H, Butrous G, Ermert L, et al. Chronic sildenafil treatment inhibits monocrotaline-induced pulmonary hypertension in rats. Am J Respir Crit Care Med. 2004;169(1):39-45.

17. Lepore JJ, Maroo A, Bigatello LM, Dec GW, Zapol WM, Bloch $\mathrm{KD}$, et al. Hemodynamic effects of sildenafil in patients with congestive heart failure and pulmonary hypertension: combined administration with inhaled nitric oxide. Chest. 2005;127(5):1647-53.

18. Zhao L, Mason NA, Morrell NW, Kojonazarov B, Sadykov A, Maripov A, et al. Sildenafil inhibits hypoxia-induced pulmonary hypertension. Circulation. 2001;104(4):424-8.

19. Lyons JM, Duffy JY, Wagner CJ, Pearl JM. Sildenafil citrate alleviates pulmonary hypertension after hypoxia and reoxygenation with cardiopulmonary bypass. J Am Coll Surg. 2004;199(4):607-14.

20. Ghofrani HA, Osterloh IH, Grimminger F. Sildenafil: from angina to erectile dysfunction to pulmonary hypertension and beyond. Nat Rev Drug Discov. 2006;5(8):689-702.

21. Guazzi M, Samaja M, Arena R, Vicenzi M, Guazzi MD. Long-term use of sildenafil in the therapeutic management of heart failure. $\mathrm{J}$ Am Coll Cardiol. 2007;50(22):2136-44.

22. Penaloza D, Arias-Stella J. The heart and pulmonary circulation at high altitudes: healthy highlanders and chronic mountain sickness. Circulation. 2007;115(9):1132-46.

23. Gorter TM, van Melle JP, Hillege HL, Pieper PG, Ebels T, Hoendermis ES, et al. Ventricular remodelling after pulmonary valve replacement: comparison between pressure-loaded and volume-loaded right ventricles. Interact Cardiovasc Thorac Surg. 2014;19(1):95-101.

24. Borgdorff MA, Bartelds B, Dickinson MG, van Wiechen MP, Steendijk P, de Vroomen M, et al. Sildenafil treatment in established right ventricular dysfunction improves diastolic function and attenuates interstitial fibrosis independent from afterload. Am J Physiol Heart Circ Physiol. 2014;307(3):H361-9.

25. Andersen S, Nielsen-Kudsk JE, Vonk Noordegraaf A, de Man FS. Right ventricular fibrosis. Circulation. 2019;139(2):269-85.

26. Crnkovic S, Egemnazarov B, Damico R, Marsh LM, Nagy BM, Douschan P, et al. Disconnect between fibrotic response and right ventricular dysfunction. Am J Respir Crit Care Med. 2018. https:// doi.org/10.1164/rccm.201809-1737OC.

Publisher's Note Springer Nature remains neutral with regard to jurisdictional claims in published maps and institutional affiliations. 